\title{
Esclerodermia localizada: a propósito de un caso clínico
}

\author{
Localized scleroderma: About a case
}

\author{
Sharon Imbett ${ }^{1}$, Carolina Suárez ${ }^{2}$, Alfonso González ${ }^{3}$ \\ 1. Médica general, residente de Pediatría, Instituto Nacional de Pediatría, Ciudad de México, México \\ 2. Médica general, Universidad de Antioquia, Medellín, Colombia \\ 3. Médico pediatra, Hospital Universitario de Sincelejo. Docente, Universidad de Sucre, Sincelejo, Colombia
}

\section{RESUMEN}

Se presenta el caso de una paciente de 4 años, quien, desde el primer año de vida, presenta una mácula hipercrómica en la región malar derecha, acompañada de retracción de la piel; el diagnóstico fue de esclerodermia localizada o morfea. Además, se hace una breve revisión bibliográfica sobre la esclerodermia localizada y sus variantes, con el objetivo de contribuir al conocimiento desde los primeros niveles de atención, y favorecer un diagnóstico oportuno, que impacte positivamente en el manejo y el pronóstico de esta enfermedad.

Se puede concluir que la esclerodermia localizada, o morfea, es una entidad que afecta la calidad de vida de quien la padece, dado que no se limita a su diversidad de manifestaciones cutáneas, sino que, en algunas variantes, presenta compromiso extradérmico. El diagnóstico temprano permite establecer un tratamiento y un seguimiento adecuados, evitando el desarrollo de complicaciones.

PALABRAS CLAVE: dermis, morfea, esclerodermia localizada, hemiatrofia facial.

\section{SUMMARY}

We present the case of a 4-year-old patient, with the appearance of a hyperchromic macula in the right region malar with skin retraction, since the first year of life. The clinicopathological correlation suggested the diagnosis of localized scleroderma or morphea. We also present a brief review of the literature about localized scleroderma and its variants, with the objective of contributing to the knowledge of the first levels of care and favoring the timely diagnosis and a positive impact in the management and prognosis of this sickness.

In conclusion, the localized scleroderma or morphea is a disease that affects the quality of life of the patient, since it is not limited to the skin with a spectrum of cutaneous manifestations, but also some compromise variants extradermic. As an early diagnosis, allows to establish an appropriate treatment and follow-up avoiding the development of complications.

KEY WORDS: Dermis, morphea, localized scleroderma, facial hemiatrophy.

\section{Correspondencia:}

Carolina Suárez Acosta

Email:

carolina.suarez@udea.edu.co

Recibido: 23/06/17

Aceptado: 02/08/18

Conflictos de interés:

No se reportan conflictos de interés.

Financiación:

Ninguna. 


\section{PRESENTACIÓN DEL CASO}

Se trata de una niña de 4 años, escolarizada, que fue llevada al Servicio de Consulta Externa Pediátrica del Hospital Universitario de Sincelejo, por presentar un cuadro clínico progresivo, que se inició en el primer año de vida y llevaba más de tres años de evolución. Consistía en la aparición de una placa hipercrómica en la región malar derecha, con retracción de la piel circunscrita, sin compromiso extracutáneo. Refirieron haber consultado en distintas ocasiones y recibir tratamiento con esteroides tópicos, sin ninguna mejoría. No había antecedentes personales ni familiares de importancia.

En el examen físico, se observó una única placa hipercrómica en la mejilla derecha, de 5 x $3 \mathrm{~cm}$, aproximadamente, de color pardo y de consistencia firme, adherida a los planos profundos. Se extendía desde la fosa lacrimal hasta el surco labiomentoniano del mismo lado, produciendo retracción de la hemicara y desviación de la región mandibular derecha y de la lengua hacia el lado afectado (figura 1).
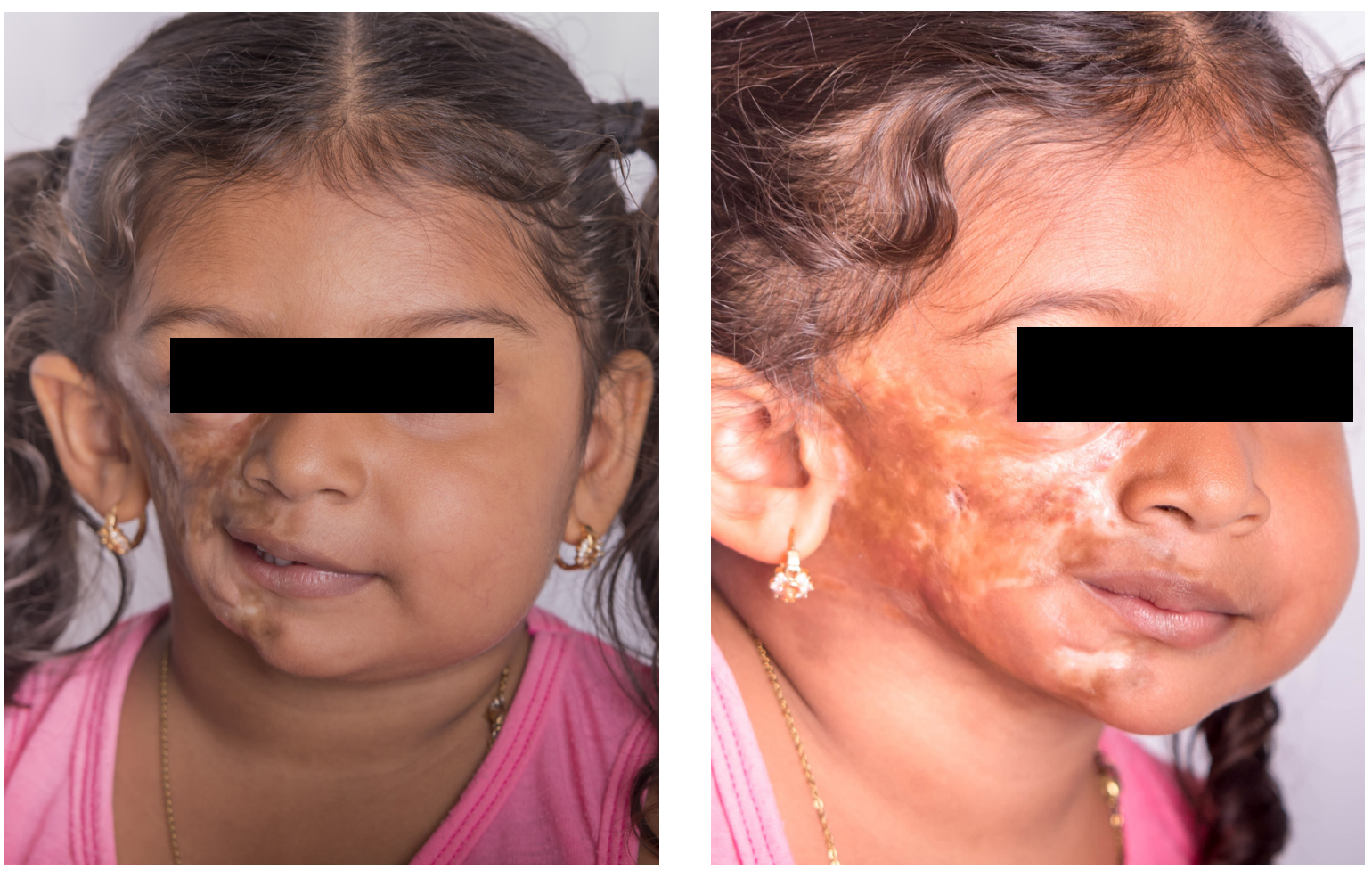

Figura 1. Esclerosis localizada cutánea (morfea). Placa fibrótica hiperpigmentada en mejilla derecha con retracción de hemicara y desviación de lengua hacia lado afectado. Hospital Universitario de Sincelejo, Sucre. 2016 *Foto realizada previo consentimiento informado de la madre y asentimiento de la paciente
En la biopsia por incisión se reportó “dermis adelgazada con leve hiperqueratosis, haces de colágeno engrosados en forma paralela a la epidermis, que atrapan a las glándulas ecrinas, muchas de ellas atróficas; además, hay focos de infiltrado inflamatorio superficial y el colágeno engrosado se extiende hasta la hipodermis”.

En los exámenes de laboratorio no se encontró alteración hematológica, ni elevación de la velocidad de sedimentación globular (VSG) o de la proteína C-reactiva (PCR). Los anticuerpos antinucleares (ANA), los antihistonas (AHA), los anti-Smith (anti-Sm) y los anticuerpos contra el ADN de doble cadena (anti-dsDNA) fueron negativos.

Con estos datos, se hizo una correlación clinicohistológica y se confirmó el diagnóstico de esclerodermia localizada profunda. 


\section{DISCUSIÓN}

La esclerodermia es una enfermedad autoinmunitaria del tejido conjuntivo, que incluye diversas entidades clínicas que se caracterizan por esclerosis dérmica, debida a la acumulación de colágeno ${ }^{(1)}$. Se presenta en distintas etapas de la vida y puede afectar exclusivamente la piel y el tejido subcutáneo. Cuando se extiende a estructuras más profundas, puede ser localizada o sistémica; de las dos anteriores, la forma localizada o morfea es más frecuente en la edad pediátrica y puede afectar la fascia, el músculo y el tejido óseo, mientras que la esclerosis sistémica afecta órganos internos y al endotelio ${ }^{(2)}$, y no será detallada en la presente revisión por no estar entre sus objetivos.

En la edad pediátrica, la esclerodermia localizada o morfea es la tercera enfermedad reumatológica más frecuente, después de la artritis juvenil idiopática y del lupus eritematoso sistémico. A nivel mundial, se estima una incidencia anual de 1 a 3 por cada 100 ooo niños, con una edad promedio de inicio entre los 7,3 y los 8,8 años, y retraso diagnóstico entre 1,2 y 1,6 años. No hay una clara evidencia de predilección racial, aunque algunos reportes indican que, en caucásicos, parece ser mayor su prevalencia, que corresponde al 70\%-82\% de los pacientes atendidos, y en una proporción de mujer a hombre de 2 a $1^{(3,4)}$.

En Colombia, existe un evidente subregistro. Según la Asociación Colombiana de Esclerodermia (ASCLER), los casos alcanzan cerca de 400o, pero, infortunadamente, no se cuenta con una estimación confiable de los que padecen esta enfermedad. Existen algunos reportes de casos y solo un estudio de caracterización de un grupo de pacientes colombianos con esclerodermia localizada juvenil, de Arango, et al. ${ }^{(2)}$. En este último, se encontró más frecuentemente en el sexo femenino, con una relación de 2,1:1. La edad promedio de inicio de la enfermedad fue de 7,1 años en la población pediátrica y el promedio de duración de la enfermedad al momento del diagnóstico fue de 16,5 meses, datos similares a los reportados en la literatura científica mundial $^{(2,3)}$.

\section{Clasificación y clínica}

Se han descrito diferentes clases de esclerodermia localizada o morfea, que comprenden distintos subtipos, los cuales están determinados por el patrón y la profundidad de la lesión ${ }^{(4,5)}$. A continuación, se detallan los subtipos de acuerdo con la clasificación provisional de la Paediatric Rheumatology European Society.

\section{Esclerosis lineal}

Corresponde al 50\%-60\% de los casos de esclerodermia localizada en niños y es la forma más frecuente en la edad pediátrica. Clínicamente, se caracteriza por bandas lineales que afectan la dermis, el tejido subcutáneo, los tendones o los huesos de extremidades, tronco o cabeza ${ }^{(6)}$.

Además, esta variante se subdivide en tres formas, según sus manifestaciones clínicas:

- $\quad$ Morfea lineal: compromete las extremidades y se asocia a contracturas y discrepancia de la longitud de las extremidades en pacientes pediátricos, pues puede afectar las placas epifisiarias de crecimiento ${ }^{(5-7)}$.

- Morfea en golpe de sable: se presenta en el 25\%, aproximadamente, de aquellos afectados por esclerodermia lineal, que tienen afectación en cuero cabelludo; se ha denominado "golpe de sable" debido a la depresión frontal paramedial que se observa ${ }^{(8)}$.

- Hemiatrofia facial progresiva: es una variante que lleva a hemiatrofia de la cara por afectación del maxilar inferior, la lengua, el tejido celular subcutáneo y el músculo, con la consecuente deformidad facial. También se conoce como síndrome de Parry-Romberg, que, al igual que la variedad en golpe de sable, se puede acompañar de alteraciones oculares, como ptosis, síndrome de Horner o endoftalmos, entre otros ${ }^{(8,9)}$. Esta variante de Parry-Romberg es la que se presenta en esta paciente. Esta entidad involucra dermatomas que afectan una o varias ramas del nervio trigémino, por lo cual los hallazgos clínicos no se limitan solo a los cambios estructurales faciales, sino que también puede presentar manifestaciones neurológicas, oculares, cutáneas y dentarias.

En cuanto a las complicaciones neurológicas, la epilepsia focal es la manifestación cerebral más común y, en algunos pacientes, se asocia a hemiatrofia cerebral con convulsiones focales intratables. También puede aparecer migraña y dolor facial por neuralgia del trigémino, entre otras. Estas complicaciones pueden asociarse a anormalidades cerebrales del mismo lado de la atrofia facial, aunque raramente puede estar afectado el hemisferio cerebral contralateral ${ }^{(9,10)}$. 


\section{Esclerosis localizada circunscrita o en placas}

Es la segunda en frecuencia y la forma más benigna; se limita, generalmente, a la dermis y, ocasionalmente, afecta el tejido adiposo subcutáneo. Se caracteriza por ser una placa ovalada y circunscrita, con áreas de induración, pigmentación o halo violáceo, que varía de acuerdo con las siguientes fases: una inicial inflamatoria y eritematosa, una indurada esclerótica y una fase atrófica asociada a hipopigmentación o hiperpigmentación ${ }^{(3-6)}$.

Los hallazgos histológicos de la primera fase consisten en infiltrado linfocitario perivascular con células plasmáticas y eosinófilos en la dermis reticular, acompañado de bandas colágenas escasas; en etapas más avanzadas, se observa predominio de las bandas colágenas, que reemplazan el infiltrado inflamatorio previo, causando atrofia y disminución de la vascularización del tejido ${ }^{(3-7)}$.

\section{Esclerosis generalizada}

Es una variante inusual; ocurre en menos del 10\% de los pacientes. Se caracteriza por afectación dérmica de placas induradas, mal definidas, ligeramente inflamadas, de localización preferente en el tronco, aunque puede afectar las extremidades y respetar la cara y las manos. En algunas ocasiones, se asocia a síntomas sistémicos inespecíficos y leves. Las pruebas inmunológicas para autoanticuerpos pueden ser positivas, especialmente los ANA, los anti-ADN y los anticuerpos antifosfolípido; además, se asocia a hipocomplementemia y eosinofilia periférica, razón por la cual se dificulta el diagnóstico diferencial con esclerosis sistémica (5-7). El fenómeno de Raynaud, la esclerodactilia y los cambios capilares en el lecho ungular son características clínicas que se presentan solo en la esclerosis sistémica, lo que permiten distinguir estas dos entidades (8-11).

\section{Panesclerótica de la infancia}

Esta variante se presenta de forma característica y predominante durante la infancia. Es la variante menos frecuente de morfea; sin embargo, es la forma que tiene el peor pronóstico, debido a su curso larvado e incapacitante y a su poca mejoría con el tratamiento. Es la morfea que causa el mayor compromiso y el más extenso; afecta la piel, el tejido celular subcutáneo y estructuras más profundas, como tendones, músculos y huesos. Se asocia a una gran morbilidad, por la atrofia muscular, las contracturas articulares y las úl- ceras secundarias. Además, se ha asociado al riesgo de desarrollar carcinoma escamocelular sobre las placas panescleróticas ${ }^{(12,13)}$.

\section{Mixta}

Se presenta hasta en el 15\% de los casos de morfea. Se caracteriza por la combinación de dos o más de las variantes anteriormente descritas. Su clínica y su pronóstico dependen del grado de afectación cutánea ${ }^{(6)}$.

\section{Diagnóstico y evaluación}

El diagnóstico de esclerodermia localizada es fundamentalmente clínico y se establece por la apariencia de las lesiones. No obstante, dado el patrón insidioso y la falta de caracterización clínica en nuestro entorno, se emplean herramientas diagnósticas; la biopsia de piel es el método de referencia para su confirmación y caracterización ${ }^{(14)}$.

A pesar de que en la mayoría de los casos no suele presentarse compromiso sistémico, es importante practicar algunos exámenes de laboratorio, como biometría hemática, química sanguínea, uroanálisis y pruebas de función renal, los cuales, generalmente, son normales. Además, buscar marcadores de inflamación sistémica, como VSG, PCR y factor reumatoideo. Estos se encuentran con mayor frecuencia en casos de esclerodermia local con manifestaciones extracutáneas, en comparación con aquellos con compromiso cutáneo exclusivo; en particular, el factor reumatoideo es positivo en aquellos que se inició con artritis ${ }^{(13,14)}$.

En cuanto a las características serológicas, hay hallazgos de autoinmunidad en algunos casos de esclerosis localizada. Esto se demostró en el estudio de Takehara y Sato ${ }^{(15)}$, quienes encontraron que los anticuerpos antinucleares en patrón homogéneo y los anticuerpos antitopoisomerasa eran los más frecuentes (73\% y 78\%, respectivamente). Los anticuerpos antinucleares son el marcador serológico clásico en casos de morfea con títulos altos, que se asocian a enfermedad temprana y manifestaciones extracutáneas ${ }^{(15)}$. A su vez, los anti-ADN de cadena sencilla (ss-DNA) son positivos en el $20 \%$ a $55 \%$ de los pacientes, y se asocian con esclerodermia lineal, afectación muscular profunda y extensión en diferentes sitios anatómicos. En contraste, los anti-dsDNA rara vez son positivos ${ }^{\left({ }^{(6)}\right.}$. Los anticuerpos antihistonas se encuentran positivos en el $47 \%$ y sus títulos altos se relacionan con aumento de la superficie corporal afectada, el número de lesiones y la aparición de contracturas articulares ${ }^{(15,17)}$. 
Los anteriores hallazgos de anticuerpos, sumados al aumento de casos de enfermedades autoinmunitarias en pacientes con esclerodermia focal en comparación con la población general, han llevado a considerar que la esclerodermia localizada se trata de una condición autoinmunitaria, que puede manifestarse simultáneamente con otras enfermedades, como la artritis juvenil idiopática, el lupus eritematoso sistémico, la psoriasis y el síndrome de Sjögren ${ }^{(13)}$.

\section{Diagnóstico diferencial}

La principal enfermedad con la que se debe hacer el diagnóstico diferencial es la esclerosis sistémica, dado que, como se mencionó anteriormente, en algunas variantes de la esclerodermia localizada, puede haber manifestaciones de compromiso sistémico y las pruebas de autoanticuerpos pueden ser positivas. Una vez descartada la esclerosis sistémica y según la variedad de esclerodermia, se pueden considerar otros diagnósticos: en la morfea circunscrita, liquen escleroso extragenital y acrodermatitis crónica atrófica; en la morfea lineal, la paniculitis lúpica; y en la morfea generalizada, fibrosis sistémica nefrogénica, esclerosis sistémica y enfermedad crónica de injerto contra huésped $^{(13,15,17)}$.

\section{Tratamiento}

El tratamiento de la esclerodermia juvenil localizada ha representado un reto para los médicos tratantes $\mathrm{y}$, hasta la fecha, se han propuesto muchas alternativas. Sin embargo, en ningún estudio controlado se ha establecido claramente su eficacia y no hay un tratamiento de referencia para esta enfermedad. Se acepta que el enfoque terapéutico depende del subtipo de esclerodermia localizada, de la afectación de los diferentes tejidos, huesos o articulaciones, de la localización y del estado de actividad de la enfermedad ${ }^{(18)}$. A continuación, se abordan las tres opciones básicas de tratamiento: el tópico, el sistémico (metotrexato, esteroides) y el tratamiento con micofenolato de mofetilo, adicionalmente de otras opciones terapéuticas aún en desarrollo.

Ante lesiones únicas, circunscritas y con poca afectación estética, son apropiados los medicamentos tópicos, como emolientes, esteroides tópicos en bajas concentraciones, tacrolimús al o,1\%, imiquimod o calcipotriol $^{(18,19)}$. Kroft, et al. ${ }^{(20)}$, en 2009, informaron sobre 10 pacientes con la variante de morfea circunscrita o en placas, activa en quienes se evaluó la eficacia del tacrolimús tópico al o,1\%, aplicado dos veces al día, durante
12 semanas. Al finalizar el tratamiento, se observó una significativa reducción de la induración de las lesiones tempranas. Estos resultados sugieren que el tacrolimús tópico puede ser una opción terapéutica en los casos de morfea activa circunscrita.

Los esteroides sistémicos, utilizados por sus efectos inmunomoduladores y antiinflamatorios, son otra alternativa terapéutica ${ }^{(18)}$. Sin embargo, Joly, et al. evaluaron la eficacia de la monoterapia en 17 pacientes y reportaron mejoría ${ }^{(21)}$, aunque su estudio fue limitado, ya que no se evaluaron las recaídas después de suspender el tratamiento. Así mismo, Bretèque, et al. (22), en un estudio retrospectivo donde se evaluaron las recaídas en adultos, obtuvieron resultados similares; la mitad de los pacientes las presentaron después de suspender el tratamiento. Esto indica la necesidad de una terapia coadyuvante, como el metotrexato.

Se recomienda el tratamiento sistémico cuando la esclerodermia localizada es de moderada a grave. En este caso, el medicamento de elección es el metotrexato, cuya dosis recomendada es de $15 \mathrm{mg} / \mathrm{m}^{2}$, administrado en una dosis semanal única (máximo, $25 \mathrm{mg}$ a la semana), durante un tiempo mínimo de 1 año. Durante los primeros 3 meses de tratamiento, se añade un curso diario de esteroides (prednisona, $1 \mathrm{mg} / \mathrm{kg}$ ) ${ }^{(23)}$. El metotrexato influye en forma importante en la cascada de efectos secundarios a la expresión de la interleucina 1 (IL-1), la IL-6 y el factor de necrosis tumoral. En la morfea y en la esclerosis sistémica, se ha visto aumento de las concentraciones séricas de estas citocinas, las cuales disminuyen paralelamente con la mejoría clínica, después de iniciar la administración de metotrexato ${ }^{(22,23)}$.

Si este esquema terapéutico falla, la tercera opción es el micofenolato de mofetilo, en dosis de 500 a 1000 $\mathrm{mg} / \mathrm{m}^{2}$. Este produce una inhibición específica de la proliferación de linfocitos T y B; además, disminuye la expresión del colágeno de tipo I, con lo cual se potencia la expresión de MMP-1 (enzima disminuida en los procesos de fibrosis) y se altera la migración de los fibroblastos ${ }^{(24)}$.

Se ha mencionado la vitamina D y sus análogos en algunos estudios. No obstante, en el único ensayo controlado, no se demostraron resultados superiores a los del placebo ${ }^{(19)}$.

La terapia de luz ultravioleta (UV) con agentes químicos, como los psoralenos o sin ellos, ha arrojado 
resultados satisfactorios en lesiones localizadas o superficiales, en algunos estudios. Los resultados de la fototerapia con UVA 1 han sido satisfactorios en condiciones in vitro en casos de esclerodermia, por reducir la síntesis de procolágeno y aumentar la expresión de colagenasa $1^{(25)}$.

En el tratamiento del síndrome de Parry-Romberg, variante localizada de la esclerodermia, primero se pretende detener la enfermedad y luego mejorar algunos síntomas. En algunos pacientes, especialmente si se afecta el sistema nervioso central, se pueden considerar medicamentos inmunosupresores, como el metotrexato más pulsos de esteroides orales a las dosis previamente mencionadas, sumados a la terapia local con emolientes, análogos de la vitamina D y fototerapia ${ }^{(18)}$. Una vez que se ha logrado inactivar la enfermedad y finalice el crecimiento del niño, se procede a restaurar la simetría facial, a través de cirugía plástica con métodos de reconstrucción entre las que se encuentra la utilización de injerto autólogo de grasa y la utilización de implantes de polietileno con lipotransferencia; injertos con transferencia microquirúrgica de tejido fascioadiposo extraído de la cara anterolateral del muslo, el serrato anterior o la zona toracodorsal, o mediante el método de regeneración volumétrico con trasplante autólogo de grasa y aplicación de ácido poli-L-láctico. Estas técnicas quirúrgicas son consideradas por algunos cirujanos como el pilar del tratamiento de la hemiatrofia facial. Cualquiera que sea la opción escogida, con estas modalidades terapéuticas se recupera solo temporalmente la buena apariencia $y$, con el paso de los años, es necesario reintervenir. Además, se pueden presentar problemas ortopédicos, que requieren cirugía correctiva ${ }^{(18,26)}$.

\section{CONCLUSIÓN}

La esclerodermia no es una enfermedad exclusivamente cutánea. Ante su diagnóstico, se debe prestar atención a la posible afectación sistémica y la asociación a otras enfermedades reumatológicas.

En pediatría, es difícil su evaluación, ante la heterogeneidad de las manifestaciones y la dificultad para predecir las secuelas o la regresión espontánea. En la consulta inicial, el médico pediatra debe tener un alto grado de sospecha para referir al paciente de forma oportuna, en primera instancia, al especialista en reumatología y al de dermatología y, de acuerdo con las manifestaciones clínicas, involucrar otras especia- lidades, como cirugía plástica, ortopedia o fisiatría, entre otras.

Queda mucho por investigar en relación con los nuevos tratamientos dermatológicos, los inmunosupresores y los productos biológicos que puedan tener una aceptable tolerancia sistémica, y aminorar las secuelas estéticas y funcionales en los niños con síndrome de Parry-Romberg.

\section{AGRADECIMIENTOS}

Los autores desean expresar sus agradecimientos a la paciente y sus familiares, al Hospital Universitario de Sincelejo y a los profesionales que colaboraron con la revisión crítica del documento final.

\section{REFERENCIAS}

1. Zulian F, Cuffaro G, SperottoF. Scleroderma in children: an update. Curr Opin Rheumatol. 2013;25(5):643-50.

2. Arango C, Malagón C, Gómez M, Mosquera C, Yépez R, González T, et al. Esclerodermia localizada juvenil: ¿es una enfermedad benigna? Rev Colomb Reumatol. 2017;24(3):129-96. http:// dx.doi.org/10.1016/j.rcreu.2017.02.006.

3. Fett N, Werth VP. Update on morphea: part I. Epidemiology, clinical presentation, and pathogenesis. J Am Acad Dermatol. 2011;64(2):217-28; quiz 229-30.

4. Torok K. Pediatric scleroderma: systemic or localized forms. Pediatr Clin North Am. 2012;59(2):381405.

5. Laxer RM, Zulian F. Localized scleroderma. Curr Opin Rheumatol. 2006;18(6):606-13.

6. Yaqub A, Chung L, Rieger KE, Fiorentino DF. Localized cutaneous fibrosing disorders. Rheum Dis Clin North Am. 2013;39(2):347-64.

7. Bielsa Marsol I. Update on the classification and treatment of localized scleroderma. Actas Dermosifiliogr. 2013;104(8):654-66.

8. Tollefson MM, Witman PM. En coup de sabre morphea and Parry-Romberg syndrome: A retrospective review of 54 patients. J Am Acad Dermatol. 2007;56(2):257-63.

9. Chiu YE, Vora S, Kwon EK, Maheshewari M. A significant proportion of children with morphea en coup de sabre and Parry-Romberg syndrome have neuroimaging findings. Pediatr Dermatol. 2012;29(6):738-48. 
10. Li SC. Scleroderma in Children and Adolescents: Localized Scleroderma and Systemic Sclerosis. Pediatr Clin North Am. 2018;65(4):757-81.

11. Leitenberger JJ, Cayce RL, Haley RW, AdamsHuet B, Bergstresser PR, Jacobe HT. Distinct autoimmune syndromes in morphea: a review of 245 adult and pediatric cases. Arch Dermatol. 2009;145(5):545-50.

12. Galán M, Rodríguez A, Vázquez MC, Jiménez R, Collantes E, Moreno JC. Morfea panesclerótica discapacitante de la infancia: un nuevo caso. Med Cutan Iber Lat Am. 2009;37:175-9.

13. Zulian F, Vallongo C, Woo P, Russo R, Ruperto N, Harper J, et al. Localized scleroderma in childhood is not just a skin disease. Arthritis Rheum. 2005;52(9):2873-81.

14. Lis-Święty A, Janicka I, Skrzypek-Salamon A, Brzezińska-Wcisło L. A systematic review of tools for determining activity of localized scleroderma in paediatric and adult patients. J Eur Acad Dermatol Venereol. 2017;31(1):30-7.

15. Takehara K, Sato S. Localized scleroderma is an autoimmune disorder. Rheumatology. 2005;44(3):274-9.

16. Takehara K, Kikuchi K, Soma Y, Igarashi A, Ishibashi Y. Anti-single-stranded DNA antibody and muscle involvement in localized scleroderma. Arch Dermatol. 1990;126(10):1368.

17. Sato S, Fujimoto M, Ihn H, Kikuchi K, Takehara K. Clinical characteristics associated with antihistone antibodies in patients with localized scleroderma. J Am Acad Dermatol.1994;31(4):567-71.

18. Careta MF, Romiti R. Localized scleroderma: clinical spectrum and therapeutic update. An Bras Dermatol. 2015;90(1):62-73.

19. Hulshof MM, Bouwes Bavinck JN, Bergman W, Masclee AA, Heickendorff L, Breedveld FC, et al. Doubleblind, placebocontrolled study of oral calcitriol for the treatment of localized and systemic scleroderma. J Am Acad Dermatol. 2000;43(6):1017-23.

20. Kroft EB, Groeneveld TJ, Seyger MM, de Jong EM. Efficacy of topical tacrolimus $0.1 \%$ in active plaque morphea: randomized, double-blind, emollient-controlled pilot study. Am J Clin Dermatol. 2009;10(3):181-7.

21. Joly P, Bamberger N, Crickx B, Belaich S. Treatment of severe forms of localized scleroderma with oral corticosteroids: follow-up study on 17 patients. Arch Dermatol. 1994;130(5):663-4.

22. Amy de la Bretèque AM, Rybojad M, Cordoliani F, Petit A, Juillard C, Flageul B, et al. Relapse of severe forms of adult morphea after oral corticosteroid treatment. J Eur Acad Dermatol Venereol. 2013;27(9):1190-1.

23. Zulian F, Vallongo C, Patrizi A, Belloni-Fortina A, Cutrone M, Alessio M, et al. A longterm followup study of methotrexate in juvenile localized scleroderma (morphea). J Am Acad Dermatol. 2012;67(6):1151-6.

24. Martini G, Ramanan AV, Falcini F, Girschick H, Goldsmith DP, Zulian F. Successful treatment of severe or methotrexate-resistant juvenile localized scleroderma with mycophenolate mofetil. Rheumatology. 2009;48(11):1410-3.

25. Sunderkötter C, Kuhn A, Hunzelmann N, Beissert S. Phototherapy: a promising treatment option for skin sclerosis in scleroderma? Rheumatology. 2006;45:52-4.

26. Lott AM, Robles MB, Marrou Wr. Transferencia de grasa autóloga en esclerodermia localizada y multicéntrica. Cir Plast Iberolatinoam. 2016;42:285-92. 